\title{
Osteomyelitis in cold-stunned Kemp's ridley sea turtles (Lepidochelys kempii) hospitalized for rehabilitation: 25 cases (2008-2018)
}

\author{
Ashley L. Powell DVM \\ Kathryn A. Tuxbury DVM \\ Julie M. Cavin DVM \\ Brian A. Stacy DVM, PhD \\ Salvatore Frasca Jr VMD, $\mathrm{PhD}$ \\ Nicole I. Stacy DVM \\ Jennifer O'Sullivan Brisson DVM \\ Mauricio Solano DVM \\ Sea Rogers Williams VMD \\ Robert J. McCarthy DVM \\ Charles J. Innis VMD
}

From the Department of Pathology and Microbiology, Atlantic Veterinary College, University of Prince Edward Island, Charlottetown, PE CIA 4P3, Canada (Powell); Animal Health Department, New England Aquarium, Boston, MA 02110 (Tuxbury, Cavin, Innis); National Oceanic and Atmospheric Administration, National Marine Fisheries Service, Office of Protected Resources at University of Florida, Gainesville, FL 32611 (BA Stacy); Connecticut Veterinary Medical Diagnostic Laboratory, Department of Pathobiology and Veterinary Science, University of Connecticut, Storrs, CT 06269 (Frasca); Department of Comparative, Diagnostic, and Population Medicine, College of Veterinary Medicine, University of Florida, Gainesville, FL 32608 (NI Stacy); Massachusetts Veterinary Referral Hospital, Woburn, MA 0I80I (Brisson); Department of Veterinary Clinical Sciences, Tufts Cummings School of Veterinary Medicine, North Grafton, MA 01519 (Solano, McCarthy); and National Marine Life Center, Bourne, MA 02532 (Williams).

Address correspondence to Dr. Powell (alpowell@ upei.ca).

\begin{abstract}
OBJECTIVE
To characterize osteolytic lesions in cold-stunned Kemp's ridley sea turtles (Lepidochelys kempii) hospitalized for rehabilitation and describe methods used for the management of such lesions.
\end{abstract}

\section{ANIMALS}

25 stranded, cold-stunned Kemp's ridley sea turtles hospitalized between 2008 and 2018.

\section{PROCEDURES}

Medical records of sea turtles with a diagnosis of osteolytic lesions were reviewed retrospectively to obtain the date of diagnosis, clinical signs, radiographic findings, microbial culture results, hematologic and plasma biochemical data, cytologic and histologic findings, antimicrobial history, time to first negative culture result, treatment duration, and outcome.

\section{RESULTS}

Lesions were identified radiographically a median of 50 days after admission and were located within epiphyses or metaphyses of various appendicular joints. Lesions were associated with periarticular swelling $(n=24)$, lameness (16), lethargy (2), and hyporexia (2). Bacterial culture yielded growth of single organisms $(n=16)$, multiple organisms $(2)$, or no growth $(6)$. Significant differences in hematologic and biochemical data were detected between the times of diagnosis and convalescence. Cytologic and histologic findings characterized the lesions as osteomyelitis leading to septic arthritis. Sixteen sea turtles were managed medically, and 8 were managed medically and surgically. Surgery resulted in rapid improvement in joint mobility and overall clinical status. Most $(22 / 25[88 \%])$ sea turtles survived and were released after long-term management.

\section{CONCLUSIONS AND CLINICAL RELEVANCE}

During rehabilitation, cold-stunned Kemp's ridley sea turtles may be affected by osteomyelitis. Medical management based on antimicrobial susceptibility testing was effective for most turtles. Long term management efforts in turtles are justified by high survival rate.
K emp's ridley sea turtles (Lepidochelys kempii) exist as a single population that is among the most endangered among marine turtle species. ${ }^{1,2}$ This species is native to the northwestern Atlantic Ocean, with adults found primarily in the Gulf of Mexico and juveniles migrating along the coast of the US during the summer. ${ }^{3}$ Juveniles often become cold stunned along the northeast coast of the United States in late autumn and early winter when water temperatures decrease to $<15^{\circ} \mathrm{C}$. ${ }^{4-7}$ When found alive, stranded cold-stunned sea turtles are collected from beaches and shallow water and transferred to rehabilitation centers for medical management.

In the northeastern United States, cold-stunned sea turtles that undergo rehabilitation have an overall survival rate of $60 \%$ to $70 \%$, with most deaths occurring within the first few days of hospitalization because of physiologic derangements secondary to hypothermia. ${ }^{5-7}$ Derangements most often associated with death include marked hypercapnia, hypoxemia, acidemia, and hyperkalemia. ${ }^{8,9}$
Morbidity and deaths of hospitalized sea turtles are most commonly due to secondary pathological conditions, including bacterial and fungal pneumonia, chronic renal failure, sepsis, and osteomyelitis. ${ }^{5,10,11}$

Details of the medical management of coldstunned turtles have been described.5,6 Briefly, turtles are gradually warmed to 24 to $25^{\circ} \mathrm{C}$ over several days and treated for dehydration, cardiorespiratory depression, metabolic derangements, and concurrent pathological conditions., 5,11-13 Physical examinations and diagnostic tests are performed, and empirical antimicrobial treatment is often initiated on admission because of the high prevalence of pneumonia and other infections. ${ }^{5,6}$ Once stabilized, sea turtles may be transferred to secondary care facilities for further rehabilitation and release or may remain in rehabilitation for 6 to 8 months until local water temperatures are suitable for release.

Osteolytic lesions have been reported in sea turtles, but most descriptions include only single cases., $5,6,10,14-21$ 
Osteolytic lesions in the phalanges of sea turtles are fairly common but appear to be transient and have limited clinical impact.5,6,14 However, some individuals develop more substantial radiographic lesions of various appendicular joints, including the shoulder, elbow, hip, carpal, and tarsal joints, resulting in joint swelling, lameness, lethargy, and hyporexia., 5,10,14,15 Previous reports ${ }^{10,20,21}$ suggest that the likelihood of survival is variable, with some survivors receiving intense, long-term medical management, surgical management, or both for as long as $\mathbf{2 0}$ months. The purpose of this study was to characterize osteolytic lesions by utilizing several interrelated diagnostic modalities and to describe methods of medical and surgical management of such lesions in a population of hospitalized cold-stunned Kemp's ridley sea turtles.

\section{Materials and Methods}

Medical management of sea turtles was conducted with authorization of the US Department of the Interior Fish and Wildlife Service (permit No. TE697823) and the National Oceanic and Atmospheric Administration National Marine Fisheries Service.

\section{Case selection criteria}

Medical records for Kemp's ridley sea turtles in the care of the New England Aquarium hospital from 2008 through 2018 were reviewed retrospectively. Cases were identified by use of the following search terms: osteomyelitis, osteolysis, osteolytic, osteotomy, arthritis, remodeling, lysis, humerus, humeral, tarsus, tarsal, carpus, carpal, tibia, ulna, radius, scapula, femur, Enterococcus, Mycobacterium, and Serratia. Data were collected, including antimicrobial history, radiographic findings at admission, date of diagnosis, clinical signs at diagnosis, disease anatomic distribution, progression of radiographic and clinical disease during serial examinations, microbial (bacterial and fungal) culture results, anatomic sites of positive culture results, hematologic and plasma biochemical data, cytologic and histologic findings, time to first negative culture result, treatment type, treatment duration, time to radiographic improvement, and case outcome. Cases that involved only transient lesions of the phalanges were excluded.

While hospitalized, sea turtles were kept in filtered rehabilitation pools containing natural sea water at 24 to $25^{\circ} \mathrm{C}$ and fed daily.

\section{Diagnostic imaging}

Initial radiography was performed for all sea turtles within the first 3 days after hospital admission as part of the general patient assessment. Radiography was performed subsequently every 2 to 4 weeks for routine monitoring and serial assessment of disease conditions such as pneumonia and osteolysis. During the study period, 6 veterinary clinicians experienced in radiographic interpretation for sea turtles evaluated the radiographs and, for specific cases, consulted with board-certified veterinary radiologists. Radio- graphs were interpreted as reported for companion animals, ${ }^{22}$ which may have resulted in variability among descriptions. For purposes of this study, osteolytic lesions that appeared to worsen radiographically over time or appeared radiographically at other anatomic sites prior to resolution were characterized as progressive. Lesions were deemed to be at their most severe point when the extent of the lesions was greatest. Skeletal anatomic nomenclature used herein follows that previously described for sea turtles. ${ }^{23-25}$

Computed tomography and scintigraphy were performed at 2 veterinary referral hospitals: Cummings School of Veterinary Medicine at Tufts University and Massachusetts Veterinary Referral Hospital. Iohexol (180 mg/kg, IV) was administered as a contrast agent in some CT examinations. Scintigraphy was performed by IV administration of 111 to 370 $\mathrm{mBq}$ ( 2 to $4 \mathrm{mCi}$ ) of technetium Tc $99 \mathrm{~m}$-labeled hydroxymethylene diphosphonate.

\section{Sample collection}

Microbial culture of blood and tissue samples, tissue biopsy, cytologic evaluation, necropsy, and histologic evaluation were used to diagnose bacterial infections. Biological samples were collected when indicated on the basis of clinical signs, physical examination results, clinicopathologic results, or radiographic abnormalities. Blood samples for microbial culture were obtained after venipuncture sites were aseptically prepared with several alternating applications of sterile povidone iodine and isopropyl alcohol. One milliliter of blood was collected from an external jugular vein with a 1.5 - to $2.5-\mathrm{cm}$ (5/8- to 1 -inch), 23 to 25 -gauge needle attached to a 1 - or 3-mL sterile syringe and immediately transferred to an acid citrate dextrose blood collection tube (BBL Septi-Check BHI; Becton Dickinson \& Co) or brain-heart infusion broth (isolator tube; Wampole Laboratories) for culture. Tissue samples for microbial culture were obtained via fine-needle aspiration, bronchoalveolar lavage, or biopsy and transferred to a sterile swab sample container or sterile glass vial for transport. Culture samples were transported at 4 to $7{ }^{\circ} \mathrm{C}$ to a commercial veterinary diagnostic laboratory (Idexx Laboratories), where cultures were initiated within 6 to 18 hours after receipt. Following initial positive culture results, sites were retested in most sea turtles at approximately 2 - to 4 -week intervals to monitor response to treatment.

\section{Microbial culture and identification}

Details of the methods generally used for bacterial and fungal culture have been described. ${ }^{26}$ Samples were maintained at 25 and $35^{\circ} \mathrm{C}$ for both aerobic and anaerobic bacterial culture and at 22 to 25 ${ }^{\circ} \mathrm{C}$ for fungal culture. When indicated, subcultures were performed and various selective media were used. ${ }^{26}$ Beginning in 2015, isolates were identified by matrix-assisted laser desorption-ionization time-offlight mass spectrometry (Bruker Scientific LLC). For 
1 turtle in which Paecilomyces (Purpureocillium) sp and Mycobacterium sp were recovered from bronchoalveolar lavage samples, samples were submitted to additional laboratories (Fungal Testing Laboratory, Department of Pathology, Long School of Medicine, University of Texas; Mycobacteriology Laboratory, Advanced Diagnostic Laboratories, National Jewish Health) for further characterization, species identification, and antimicrobial susceptibility testing.

\section{Antimicrobial susceptibility testing}

Antimicrobial susceptibility of recovered isolates was determined via commercially available susceptibility cards (VITEK colorimeter; bioMérieux Inc) and Kirby-Bauer disk diffusion assay in accordance with performance standards of the Clinical and Laboratory Standards Institute. ${ }^{27}$ Selection of antimicrobials for susceptibility testing was determined by the diagnostic laboratory (Idexx Laboratories). Minimum inhibitory concentrations were determined, and isolates were classified as susceptible, intermediate, or resistant.

\section{Surgery}

Sea turtles were considered surgical candidates if they continued to have positive bacterial culture results despite several months of antimicrobial treatment, persistence of clinical signs, or progressive lysis identified on diagnostic imaging. Preoperative analgesia included meloxicam $(0.2$ to $1.0 \mathrm{mg} / \mathrm{kg}, \mathrm{IM})$ or carprofen ( 1 to $2 \mathrm{mg} / \mathrm{kg}$, IM or SC), morphine (1.0 $\mathrm{mg} / \mathrm{kg}, \mathrm{IM})$, and lidocaine (0.2 to $0.4 \mathrm{mg} / \mathrm{kg}$, locally) or bupivacaine (1 to $2 \mathrm{mg} / \mathrm{kg}$, locally). General anesthesia was induced with ketamine $(5 \mathrm{mg} / \mathrm{kg}$, IV) and dexmedetomidine $(0.05 \mathrm{mg} / \mathrm{kg}, \mathrm{IV})$, followed by endotracheal intubation and intermittent positivepressure ventilation with sevoflurane in oxygen or air at 1 to 2 breaths/min. Surgical procedures included arthrotomy, debridement, and removal of sequestrae from the affected site or sites. Debridement of necrotic material was performed with a curette, and the site was thoroughly lavaged prior to closure. Medications provided to induce anesthetic recovery included atipamezole $(0.5 \mathrm{mg} / \mathrm{kg}, \mathrm{IM})$, naloxone $(0.02$ to $0.2 \mathrm{mg} /$ $\mathrm{kg}, \mathrm{IM})$, and atropine $(0.05 \mathrm{mg} / \mathrm{kg}, \mathrm{IM})$ or epinephrine $(0.1 \mathrm{mg} / \mathrm{kg}, \mathrm{IM})$.

Radiographs of affected sites were obtained before and after surgery. After surgery, sea turtles were returned to rehabilitation pools as soon as they recovered adequately from anesthesia, often within several hours. Incisions were monitored daily, and skin sutures or staples were removed in 4 to 6 weeks.

\section{Cytologic and histologic evaluation}

Fine-needle aspirate and bronchoalveolar lavage samples were cytologically evaluated by attending veterinarians, with selected samples also evaluated by a board-certified veterinary clinical pathologist (NIS). Histologic evaluation of biopsy and bone sequestrum samples was conducted by 1 of 2 board-certified vet- erinary pathologists (BAS and SF), who used previously described methods. ${ }^{11}$ When relevant, postmortem samples were collected after euthanasia, which was achieved by administration of ketamine $(5 \mathrm{mg} / \mathrm{kg}$, IV) and dexmedetomidine $(0.05 \mathrm{mg} / \mathrm{kg}$, IV), followed by pentobarbital (100 mg/kg, IV or intracoelemically), potassium chloride ( $2 \mathrm{mEq} / \mathrm{kg}$, IV or intracoelemically), and lidocaine $[10 \mathrm{mg} / \mathrm{kg}$, intracranially).

\section{Hematologic and plasma biochemical analyses}

Hematologic and plasma biochemical analyses were conducted at a commercial veterinary diagnostic laboratory (Idexx Laboratories). Manual WBC counts were determined with a hemocytometer and phloxine B stain. Differential WBC counts were performed by a board-certified veterinary clinical pathologist, who used a blood smear stained with modified Wright-Giemsa stain. Plasma biochemical analysis was performed with 1 of 2 automated chemistry analyzers (AU5422; Olympus America; AU5432; Beckman Coulter).

\section{Statistical analysis}

Data were analyzed with statistical software (Excel 2013 version 15.0.5223.1001; Microsoft Corp). Hematologic and plasma biochemical data at the time of radiographic diagnosis were compared with convalescent data with the paired $t$ test (normally distributed data) or Wilcoxon signed rank test (nonnormally distributed data). For this purpose, convalescent data were defined as those pertaining to the last samples collected prior to release, after resolution of clinical signs and discontinuation of medical management. Normality was determined via the Jarque-Bera (skewness-kurtosis) test and $z$ test. Values of $P<0.05$ were considered significant.

\section{Results}

\section{Animals}

A total of 2,226 live, cold-stunned, juvenile Kemp's ridley sea turtles were admitted to the New England Aquarium hospital for rehabilitation from November 2008 to January 2018. At the time of admission, no osteolytic lesions were noted on radiographs. Of the 2,226 sea turtles, 25 (1.1\%) developed osteolytic lesions during rehabilitation that met the study inclusion criteria. Included sea turtles had a mean straight carapace length of $25.9 \mathrm{~cm}$ (median, $25.4 \mathrm{~cm}$; range, 21 to $34 \mathrm{~cm}$ ) and weighed a mean of $2.7 \mathrm{~kg}$ (median, $2.5 \mathrm{~kg}$; range, 1.5 to $5.9 \mathrm{~kg}$ ). Sixteen (64\%) sea turtles were managed medically, and 8 (32\%) were managed medically and surgically.

\section{Clinical and radiographic abnormalities}

Prior to and at the time of radiographic diagnosis of osteolytic lesions, abnormalities in clinical status and physical examination findings were noted in all 25 sea turtles and included periarticular swelling $(n=24)$, lameness (16), lethargy (2), and hyporexia 
(2). Initial radiographic lesions were observed after a median of 50 days of hospitalization (range, 15 to 116 days; mean $\pm \mathrm{SD}, 55 \pm 2$ days), including 1 turtle in which osteolytic lesions were diagnosed after transfer to $\mathrm{Na}$ tional Marine Life Center, Buzzards Bay, Mass. Visible swelling was noted after a median of 44 days of hospitalization (range, 1 to 103 days; mean \pm $\mathrm{SD}, 42 \pm 26$ days). Osteolytic lesions affected the humerus $(n=15[60 \%])$, radius or ulna (13 [52\%]), carpal bones (11 [44\%]), femur (5 [20\%]), tibia or fibula (5 [20\%]), tarsal bones (4 [16\%]), scapula or coracoid (4 [16\%]), ilium (1 [4\%]), and mandible (1 [4\%]). Twenty $(80 \%)$ turtles had multiple sites affected. Eleven of the 25 (44\%) turtles had unilateral lesions, with 1 joint affected on 1 half of the body, but the contralateral joint was unaffected. The remaining 14 (56\%) turtles had bilateral involvement of 1 or more joints.

Early-stage osteolytic lesions were restricted to the epiphysis and metaphysis of long bones and were characterized by a focal radiolucent region of geographic to moth-eaten lysis on radiographs (Figures I and 2 ). No lesions were detected in the diaphysis. Early stages of the disease were associated with increased periarticular soft tissue opacity and thickening, suggesting periarticular swelling and arthritis. Later stages of the disease were characterized by increased bone opacity and remodeling of lesion borders. No evidence of new bone formation was observed in regions containing larger lytic foci.

Progression between early- and latestage lesions occurred over a median of 61 days (range, 15 to 286 days; mean \pm SD, $83 \pm 62$ days; $n=23$; Table I). No progression was noted in the area occupied by the lesion and number of affected joints in 2 sea turtles.

\section{Other diagnostic imaging}

Further diagnostic imaging included CT ( 4 turtles) and scintigraphy (2 turtles). Selection of cases for advanced imaging was at the discretion of clinicians, generally with the intention of seeking further insight into complicated cases regarding surgical planning, prognosis, and decisions regarding resolution prior to release. Computed tomography revealed mostly osteolytic processes of the cortex and medulla of affected bones, with loss of subchondral bone and the
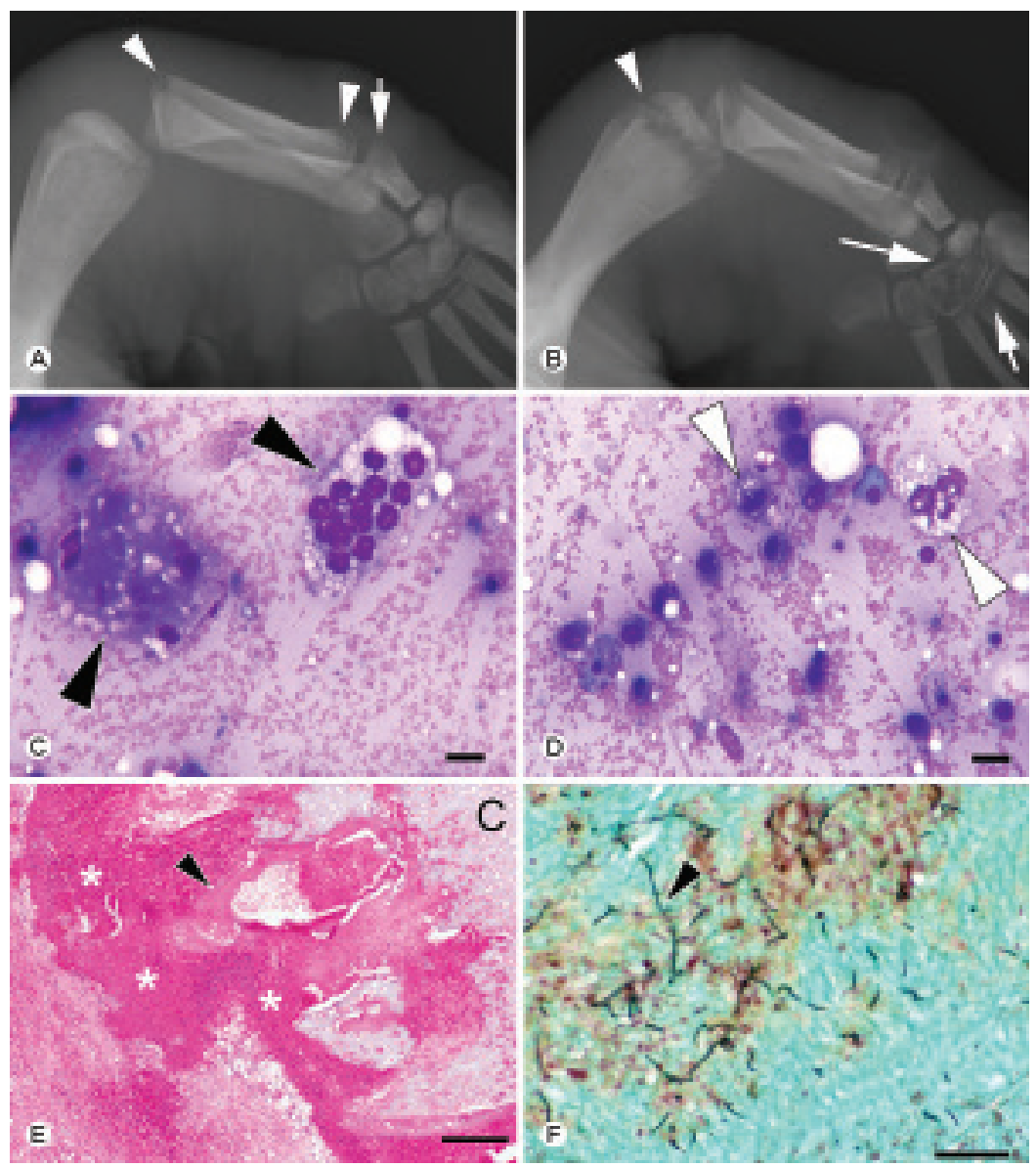

Figure I-Radiographic (A and B), cytologic (C and D), and postmortem histologic ( $E$ and $F$ ) images of osteolytic lesions in a Kemp's ridley sea turtle (Lepidochelys kempii) that developed swelling of multiple joints 2 weeks after admission to the hospital for rehabilitation. Microbial culture of blood and bone samples yielded Serratia marcescens. The turtle was euthanized 3 months after stranding because of disease progression. Results of postmortem examination suggested a systemic fungal (Metarrhizium sp) infection. A-Dorsoventral radiographic view of the left elbow joint and carpus I month after stranding showing early lysis of the distal aspect of the humerus, proximal and distal aspect of the ulna (arrowheads), intermedium (arrow), and base of metacarpal bones I to 3. B-Dorsoventral radiographic view of the same region as in panel A 2 months after stranding showing progressive lysis of the distal aspect of the humerus (arrowhead), proximal and distal aspects of the radius and ulna, intermedium, carpal bones I to 5 (long arrow), and base of metacarpal bones I to 4 (short arrow). C and D-Photomicrographs of a fine-needle aspirate sample from the affected elbow joint showing evidence of osteolysis (osteoclasts; black arrowheads; C) and marked histiocytic inflammation (white arrowheads; D). No infectious agents were observed. Wright-Giemsa stain; bar $=10 \mu \mathrm{m}$. E-Photomicrograph of a histologic section of the bone and cartilage sequestrum from the affected elbow joint showing intense heterophilic and histiocytic inflammation (asterisks) that fills the medullary spaces of the epiphysis, surrounds necrotic subchrondral bone (arrowhead), and undermines the articular cartilage. H\&E stain; bar $=240 \mu \mathrm{m}$. F-Another photomicrograph of a histologic section of the bone and cartilage sequestrum from the affected elbow joint showing septate fungal hyphae with dichotomous branching (arrowhead) within an area of osteomyelitis affecting subchondral bone. Gomori methenamine silver stain; bar $=45 \mu \mathrm{m}$.

articular surface, soft tissue swelling, and occasionally pathologic fractures. In 1 sea turtle, scintigraphy revealed focal, asymmetric radiopharmaceutical uptake of moderate intensity at the lytic sites. Intensity of radiopharmaceutical uptake varied and did not appear to be correlated with extent of lysis as noted on radiographs. In another sea turtle, scintigraphy was 

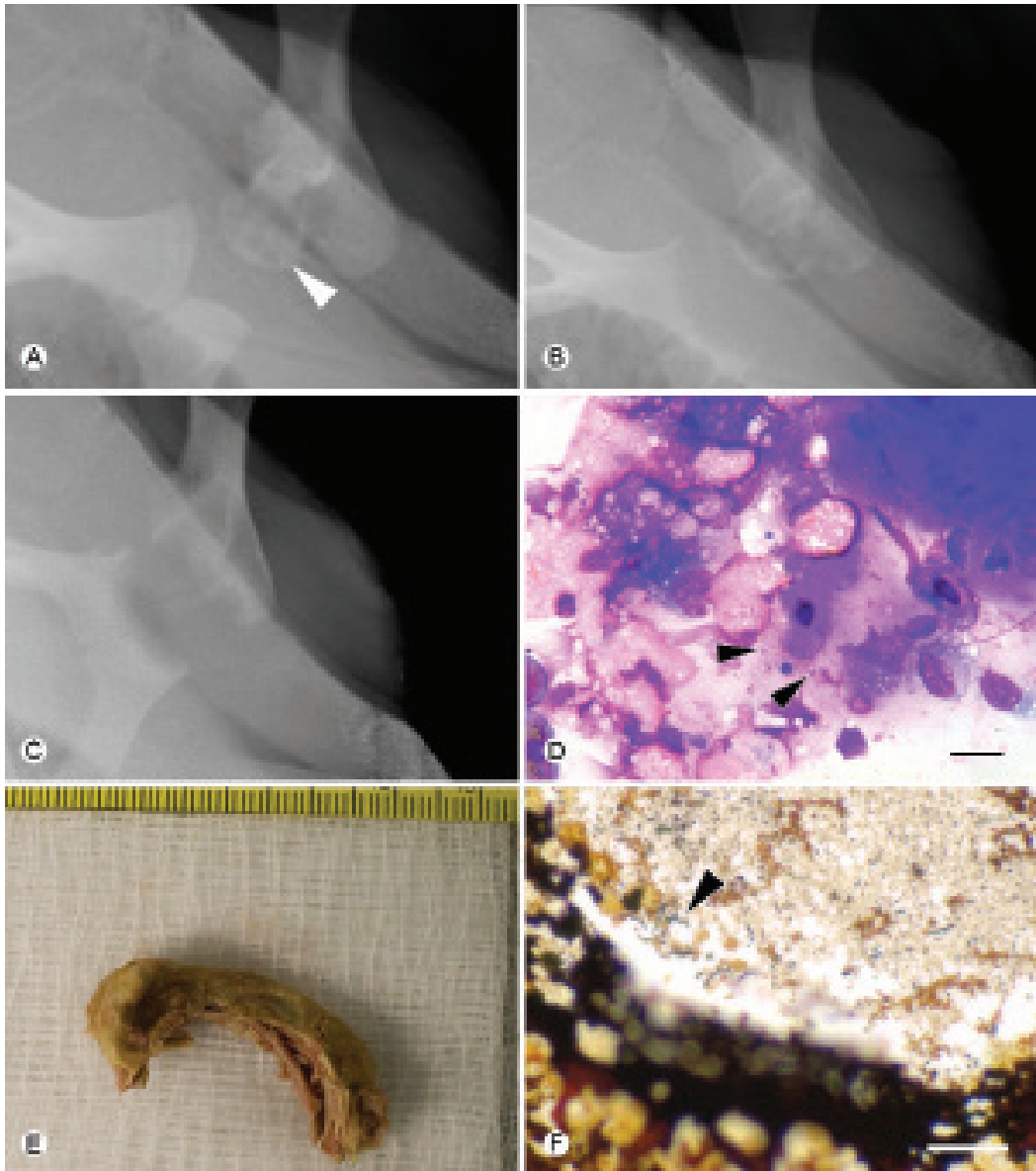

e

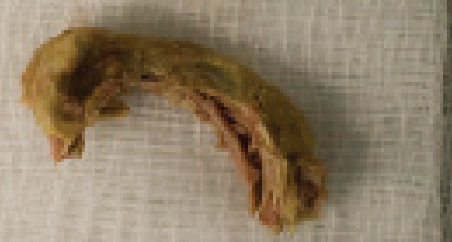

Figure 2-Radiographic, cytologic, gross, and histologic images of osteolytic lesions in a Kemp's ridley sea turtle that developed lameness of the left shoulder joint 2 months after becoming stranded. Blood culture and bone culture grew $S$ marcescens. The turtle was released to the wild after successful surgical management. A-Dorsoventral radiographic view of the left shoulder joint at the time of lameness onset showing a moderately moth-eaten appearance (osteolysis) of the humeral head (arrowhead). B-Dorsoventral radiographic view of the same region 2 months later showing progressive lysis of the humeral head, greater tubercle, and distal aspect of the scapula, and coracoid. C-Dorsoventral radiographic view of the same region 4 months after arthrotomy showing sclerotic, smooth bone margins, indicating completion of new bone formation. Major anatomic landmarks such as the humeral head and neck and the articular surfaces and subchondral bone of the glenoid fossa and coracoid are no longer present. $D$-Photomicrograph of a fine-needle aspirate sample from the left shoulder joint obtained at the time of the radiographic image in panel B showing moderate heterophilic and histiocytic inflammation with presence of extracellular monomorphic small rod-shaped bacteria (arrowheads). Romanowsky-type stain (JorVet Dip Quick Stain; Jorgensen Laboratories); bar = $10 \mu \mathrm{m}$. E-Photograph of a necrotic humeral head sequestrum removed during arthrotomy. The provided scale is in millimeters. F-Photomicrograph of a humeral head sequestrum sample showing elongated bacterial bacilli within an area of osteomyelitis affecting subchondral bone. Warthin-Starry stain; bar $=35 \mu \mathrm{m}$.

conducted during convalescence and revealed no increased radiopharmaceutical uptake.

\section{Microbial culture}

Microbial culture of fine-needle aspirate samples, intraoperative biopsy, or swab samples from osteolytic sites was performed for 24 sea turtles. No microbial culture was performed for the remaining sea turtle. Sixteen sea turtles were receiving antimicrobials at the time of culture. Bacterial culture yielded single-organ- ism growth ( $\mathrm{n}=16)$, mixed growth (2), or no growth (6). Bacteria isolated from osteolytic sites in 18 sea turtles included Enterococcus spp $(\mathrm{n}=10)$, Serratia marcescens (7), Citrobacter braakii (1), Acinetobacter baemolyticus (1), Escherichia coli (1), Morganella morganii (1), Mycobacterium chelonae (1), Pseudomonas sp (1), and Vibrio alginolyticus (1). Results indicated mixed bacterial growth for samples from 2 sea turtles. Enterococcus spp, Pseudomonas aeruginosa, and $E$ coli were initially isolated for one turtle, and culture of a second sample yielded Enterococcus spp, Pseudomonas mendocina, and C braakii. Culture results for the other turtle included $C$ braakii, Enterococcus faecalis, and $M$ morganii. For the 8 sea turtles that underwent surgery, microbial culture results for necrotic tissue samples were positive and corresponded with results for fine-needle aspirate samples. No fungi were isolated from premortem samples.

Microbial culture of blood samples was completed for 21 sea turtles. Eleven sea turtles that received such testing yielded single (vs multiple) bacterial species, including $E$ faecalis, Enterococcus spp, and $S$ marcescens. For 8 sea turtles, these results corresponded with those for the osteolytic site. In 1 sea turtle with concomitant pneumonia, a Mycobacterium sp was isolated from bone and bronchoalveolar lavage samples, and Enterococcus spp was isolated from the blood sample. The bronchoalveolar lavage isolate was further characterized as $M$ chelonae at a referral laboratory.

\section{Antimicrobial treatment and susceptibility testing}

Fifteen of $25(60 \%)$ sea turtles received ceftazidime $(22 \mathrm{mg} / \mathrm{kg}$, IM, q 3 days) on admission as initial prophylaxis, for a mean treatment duration of 41 days. The other 10 (40\%) turtles received oxytetracycline $(42 \mathrm{mg} / \mathrm{kg}$, IM or SC, q 6 days) as initial prophylaxis, for a mean treatment duration of 43 days.

Nine of 10 sea turtles with culture results indicating Enterococcus spp in blood or osteolytic site samples ( $n=13$ isolates) had received ceftazidime as initial prophylaxis. All 8 sea turtles with culture results indicating $S$ marcescens $(\mathrm{n}=9$ isolates) had received oxytetracycline as initial prophylaxis on admission to the hospital. Antimicrobial susceptibility data for Enterococcus spp and $S$ marcescens isolates were summarized (Supplementary Table SI). All Enterococcus isolates were sensitive to amoxicillin, amoxicillin-clavulanic 
Table I-Duration of various periods (days) during rehabilitation of Kemp's ridley sea turtles (Lepidochelys kempii) that developed osteolytic lesions while hospitalized.

\begin{tabular}{lcc} 
Period & Mean \pm SD & Median (range) \\
\hline Admission to initial clinical signs $(n=25)$ & $42 \pm 25.8$ & $44(0-103)$ \\
Admission to initial diagnosis $(n=25)$ & $55 \pm 23.9$ & $50(15-116)$ \\
Initial diagnosis to lesion progression $(n=23)$ & $47 \pm 44.6$ & $33(7-162)$ \\
Initial diagnosis to most severe lesion $(n=25)$ & $83 \pm 62.5$ & $61(15-286)$ \\
Initial diagnosis to surgery $(n=8)$ & $155 \pm 113.8$ & $98(75-382)$ \\
Start of initial treatment to surgery $(n=8)$ & $107.3 \pm 82.5$ & $85(0-280)$ \\
Admission to release $(n=22)$ & $270 \pm 90.6$ & $256(133-552)$ \\
Initial diagnosis to release $(n=22)$ & $213 \pm 98.5$ & $204(70-537)$ \\
Surgery to release $(n=7)$ & $162 \pm 82.4$ & $123(118-343)$
\end{tabular}

acid, and chloramphenicol with varying susceptibility to azithromycin, ciprofloxacin, doxycycline, enrofloxacin, gentamicin, marbofloxacin, and tetracycline. All $S$ marcescens isolates were sensitive to cefovecin, ceftazidime, ceftiofur, ciprofloxacin, enrofloxacin, and marbofloxacin, but resistant to cephalexin and tetracycline. There was variable susceptibility to amikacin, cefpodoxime, ceftazidime, chloramphenicol, gentamicin, and tobramycin. The Mycobacterium isolate recovered from an intraoperative bone biopsy sample from 1 sea turtle was sensitive to kanamycin, tobramycin, gentamicin, clarithromycin, azithromycin, trimethoprim-sulfamethoxazole, and tigecycline. The isolate had intermediate susceptibility to cefepime, linezolid, and moxifloxacin, and was resistant to amoxicillin/clavulanic acid, ceftriaxone, cefoxitin, ciprofloxacin, doxycycline, and minocycline. Antimicrobial susceptibility testing was also completed for single Pseudomonas sp, E coli, V alginolyticus, $C$ braakii, and $A$ haemolyticus isolates. Detailed presentation of susceptibility data for each of these isolates is beyond the scope of this report, but subsequent antimicrobial selection was based on these susceptibility results.

In addition to initial antimicrobial prophylaxis, 23 sea turtles later received empirical treatment with ceftazidime (22 mg/kg, IM, q $3 \mathrm{~d}$; median duration, 42 days), amikacin (10 mg/kg, IV or IM, q 3 d; median duration, 69 days), ampicillin (30 mg/kg, IM, q $24 \mathrm{~h}$; median duration, 97 days), amoxicillin-clavulanic acid (30 mg/kg, PO, q 24 h; median duration, 68 days), enrofloxacin ( $20 \mathrm{mg} / \mathrm{kg}$, SC or PO, q 3 $\mathrm{d}$; median duration, 48 days), gentamicin $(2.5 \mathrm{mg} / \mathrm{kg}$, IM, q 3 days; median duration, 32 days), or oxytetracycline (42 $\mathrm{mg} / \mathrm{kg}$, IM or SC, q $6 \mathrm{~d}$; median duration, 22 days). The distribution of sea turtles receiving the various antimicrobials was summarized (Figure 3). Overall, empirical antimicrobial treatment was administered for a median of 59 days (range, 15 to 200 days; mean \pm SD, $71 \pm 50$ days).

Five of the 23 (22\%) sea turtles receiving empirical antimicrobial treatment improved and did not require additional treatment. After results of microbial culture and antimicrobial susceptibility testing were obtained, 17 sea turtles received specific antimicrobial treatment and further therapeutic management, consisting of ceftazidime (median duration, 36 days), amikacin (median duration, 60 days), ampicillin (median duration, 78 days), amoxicillin/clavulanic acid (median duration, 68 days), enrofloxacin (median duration, 83 days), or gentamicin (median duration, 48 days). Specific antimicro-

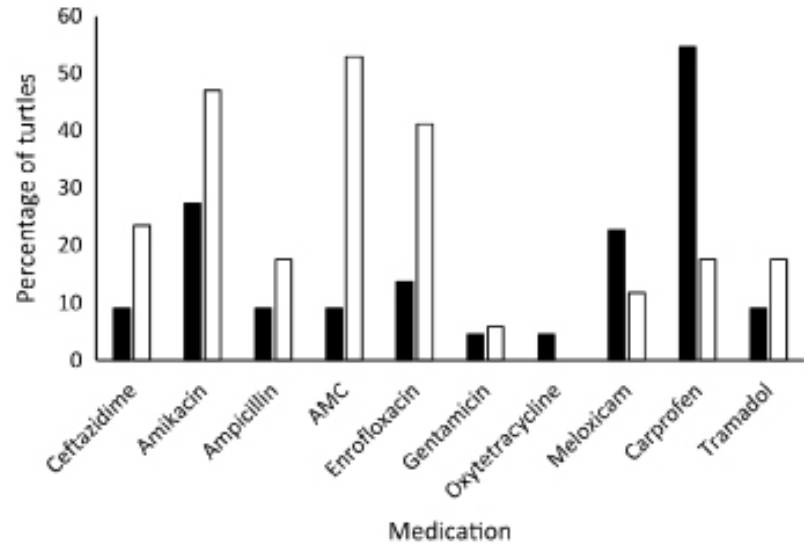

Figure 3-Medications used on an empirical basis (black; $\mathrm{n}=$ 22 ) or as additional or susceptibility-based treatment (white; 17) in the rehabilitation of 25 Kemp's ridley sea turtles with osteolytic lesions. AMC = Amoxicillin-clavulanic acid.

bial treatment and further therapeutic management was administered for a median of 85 days (range, 31 to 189 days; mean \pm SD, $98 \pm 47$ days). Fourteen of $17(82 \%)$ sea turtles improved after specific treatment.

Nineteen of 25 (76\%) sea turtles received anti-inflammatory and analgesic treatment consisting of carprofen (1 to $2 \mathrm{mg} / \mathrm{kg}$, IM, SC, or PO, q $24 \mathrm{~h}$ to q $3 \mathrm{~d}$; median duration, 60 days), meloxicam ( 0.2 to $1.0 \mathrm{mg} / \mathrm{kg}$, IM or PO, q $24 \mathrm{~h}$ to $\mathrm{q} 3 \mathrm{~d}$; median duration, 26 days), with or without tramadol (5 to $10 \mathrm{mg} / \mathrm{kg}$, PO, q $48 \mathrm{~h}$ to q $3 \mathrm{~d}$; median duration, 59 days). Anti-inflammatory medications were administered as part of initial treatment in 18 sea turtles and as part of subsequent treatment in 7 sea turtles.

\section{Surgery}

Five sea turtles underwent arthrotomy of 1 joint: either the shoulder or carpal joint. Three sea turtles required arthrotomy in multiple joints, including unilateral shoulder and bilateral elbow joints $(n=1)$, bilateral shoulder joints (1), or unilateral elbow and knee joints (1). Affected joints contained compacted caseous material and single or multiple sequestra. For 1 sea turtle, $1.5 \mathrm{~mL}$ of compounded vancomycin gel $(20 \mathrm{mg} /$ $\mathrm{mL})$ was infused into the surgical site prior to closure.

\section{Cytologic and histologic findings}

Cytologic examination of fine-needle aspirate samples was performed for 8 sea turtles. Results in- 
dicated marked inflammation with evidence of osteolysis (eg, presence of osteoclasts and heterophilic and histiocytic inflammation with or without the presence of bacteria; Figures 1 and 2). Histologic evaluation of surgically removed bone sequestra was performed for 7 sea turtles, revealing locally extensive, chronic-active, and necrotizing heterophilic and granulomatous epiphyseal osteomyelitis, with osteoclastic resorption of bone and adjacent chondronecrosis. Bone necrosis was characterized by bony spicules having necrotic osteocytes within lacunae or by bony spicules having empty lacunae. Intralesional bacteria were observed in samples from 3 sea turtles.

\section{Hematologic and plasma biochemical findings}

Hematologic and plasma biochemical results at the time of radiographic diagnosis and convalescence were available for 21 survivors (Table 2). Initial samples were collected a median of 10 days (range,
1 to 34 days; mean $\pm \mathrm{SD}, 10 \pm 9$ days) prior to radiographic diagnosis of osteolytic lesions. Convalescent data were collected a median of 22 days (range, 1 to 60 days; mean \pm SD, $25 \pm 15$ days) after completion of antimicrobial treatment, within 1 month prior to release. Compared with convalescent values, hematologic and biochemical data at the time of radiographic diagnosis were significantly higher for WBC, absolute heterophil, absolute and relative monocyte, absolute and relative basophil, and relative eosinophil counts; plasma chloride and cholesterol concentrations; and alkaline phosphatase activity. On the other hand, plasma albumin, total protein, phosphorus, and potassium concentrations were significantly lower at the time of radiographic diagnosis.

\section{Outcome}

Twenty-two of 25 (88\%) sea turtles survived to release to the wild, and 3 died or were euthanized. The 22 successfully managed sea turtles included 7

Table 2-Initial (at the time of radiographic diagnosis) and convalescent hematologic and plasma biochemical data for 21 surviving sea turtles with osteolytic lesions.

\begin{tabular}{|c|c|c|c|c|c|}
\hline \multirow[b]{2}{*}{ Variable (range) } & \multicolumn{2}{|c|}{ Initial } & \multicolumn{2}{|c|}{ Convalescent } & \multirow{2}{*}{$\begin{array}{c}\text { Reference data* } \\
\text { Mean } \pm \text { SD }\end{array}$} \\
\hline & Mean \pm SD & Median (range) & Mean \pm SD & Median (range) & \\
\hline Hct (\%) & $29.2 \pm 5.5$ & $27.5(20.5-4 I)$ & $28.9 \pm 3.0$ & $28.5(25-35)$ & $30.0 \pm 3.0(26-37)$ \\
\hline WBCs $\left(\times 10^{3} / \mu \mathrm{L}\right) \dagger$ & $9.7 \pm 6.0$ & $8.0(4-27.6)$ & $5.8 \pm 2.7$ & $5.0(1.7-10.4)$ & $4.3 \pm 2.4(1.1-10.2)$ \\
\hline Heterophils (\%) & $60.2 \pm 14.6$ & $60.0(30-90)$ & $56.3 \pm 9.5$ & $57.5(3 \mid-69)$ & $55.0 \pm$ II.0 (35-80) \\
\hline Lymphocytes (\%) & $34.3 \pm 14.2$ & $36.0(8-63)$ & $38.2 \pm 11.8$ & $36.5(15-62)$ & $35.0 \pm 11.0(15-60)$ \\
\hline Monocytes (\%)*t & $4.5 \pm 3.1$ & $3.5(1-12)$ & $2.9 \pm 2.8$ & $2.0(0-10)$ & $7.0 \pm 5.0(1-16)$ \\
\hline Eosinophils (\%)† & $0.9 \pm 1.6$ & $0.0(0-7)$ & $2.4 \pm 3.2$ & $1.0(0-11)$ & $2.0 \pm 2.0(0-6)$ \\
\hline Basophils (\%)† & $0.1 \pm 0.6$ & $0.0(0-3)$ & $0.2 \pm 0.4$ & $0.0(0-1)$ & $0.8 \pm 1.4(0-5)$ \\
\hline Heterophils $\left(\times 10^{3} / \mu \mathrm{L}\right)^{*}$ & $7.3 \pm 6.7$ & $4.9(1.5-25.64)$ & $2.9 \pm 1.3$ & $2.7(0.6-5)$ & $2.2 \pm 1.0(0.6-4.1)$ \\
\hline Lymphocytes $\left(X 10^{3} / \mu \mathrm{L}\right)$ & $3.1 \pm 2.0$ & $2.5(1.0-9.8)$ & $2.4 \pm 1.6$ & $1.6(0.6-5.6)$ & $1.7 \pm 1.5(0.3-5.6)$ \\
\hline Monocytes $\left(\times 10^{3} / \mu \mathrm{L}\right) \dagger$ & $0.5 \pm 0.7$ & $0.3(0-3.3)$ & $0.2 \pm 0.2$ & $0.1(0-5)$ & $0.3 \pm 0.2(0.09-0.7)$ \\
\hline 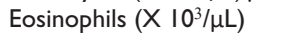 & $0.1 \pm 0.2$ & $0.0(0-0.8)$ & $0.1 \pm 0.2$ & $0.1(0-5)$ & $1.0 \pm 0.2(0.0-0.6)$ \\
\hline Basophils $\left(\times 10^{3} / \mu \mathrm{L}\right) \dagger$ & $0.01 \pm 0.04$ & $0.00(0-0.20)$ & $0.00 \pm 0.03$ & $0.00(0-0.10)$ & $0.30 \pm 0.60(0-0.20)$ \\
\hline Glucose (mg/dL) & $122.2 \pm 21.3$ & $122.5(85-|8|)$ & $121.1 \pm 13.8$ & I 19.5 (96-165) & $116 \pm 16(99-146)$ \\
\hline Creatinine (mg/dL) & $0.1 \pm 0.1$ & $0.1(0-0.4)$ & $0.0 \pm 0.04$ & $0.0(0-0.1)$ & $0.2 \pm 0.1(0-0.3)$ \\
\hline BUN (mg/dL) & $110.8 \pm 51.9$ & $124.0(|7-2| 2)$ & $1 \mid 5.5 \pm 22.3$ & II 3.5 (70-170) & $136 \pm 2 \mid(87-164)$ \\
\hline BUN:Cr ratio & $476.0 \pm 310.8$ & $460.0(210-990)$ & $877.5 \pm 167.8$ & $860.0(700-1090)$ & - \\
\hline Uric acid (mg/dL) & $1.1 \pm 1.5$ & $0.6(0.3-5.6)$ & $0.5 \pm 0.2$ & $0.5(0.3-1.2)$ & $0.5 \pm 0.2(0.2-0.9)$ \\
\hline Phosphorus (mg/dL) $\dagger$ & $7.9 \pm 1.4$ & $7.6(5.5-10.9)$ & $8.0 \pm 2.4$ & $7.7(4.8-13.7)$ & $9.0 \pm 1.7(4.9-1 \mid .7)$ \\
\hline Calcium (mg/dL) & $7.0 \pm 0.9$ & $7.3(5-8)$ & $7.4 \pm 1.3$ & $7.2(4.3-9.5)$ & $7.5 \pm 1.3(6.1-10.8)$ \\
\hline Sodium (mmol/L) & $154.0 \pm 4.6$ & $154.0(144-165)$ & $152.1 \pm 3.1$ & $153.0(144-155)$ & $154 \pm 5(|45-16|)$ \\
\hline Potassium $(\mathrm{mmol} / \mathrm{L}) \dagger$ & $3.7 \pm 0.8$ & $3.5(2.3-6.2)$ & $3.9 \pm 0.6$ & $3.7(3-5.2)$ & $3.9 \pm 0.6(3.0-5.1)$ \\
\hline $\mathrm{Na}: \mathrm{K}$ ratio & $43.5 \pm 9.2$ & $43.2(20-63)$ & $40.5 \pm 5.1$ & $41.0(29-50)$ & $40 \pm 5(32-50)$ \\
\hline Chloride $(\mathrm{mmol} / \mathrm{L}) \dagger$ & $118.0 \pm 4.6$ & $118.0(107-130)$ & $114.9 \pm 4.3$ & $115.0(107-123)$ & $116 \pm 4(109-122)$ \\
\hline $\mathrm{TCO}_{2}(\mathrm{mmol} / \mathrm{L})$ & $30.6 \pm 4.1$ & $30.8(22-38)$ & $32.1 \pm 5.6$ & $33.0(20-44)$ & - \\
\hline Anion gap (mmol/L) & $9.3 \pm 4.2$ & $9.0(I-18)$ & $9.4 \pm 5.1$ & $8.0(4-25)$ & - \\
\hline Plasma protein $(\mathrm{mg} / \mathrm{dL}) \dagger \ddagger$ & $4.2 \pm 0.6$ & $4.3(3.2-5.6)$ & $4.5 \pm 0.5$ & $4.6(3.5-5.3)$ & - \\
\hline Total protein $(\mathrm{g} / \mathrm{dL}) \dagger$ & $3.2 \pm 0.6$ & $3.2(2.3-4.3)$ & $3.6 \pm 0.3$ & $3.6(3-4.2)$ & $3.3 \pm 0.4(2.5-4.1)$ \\
\hline Albumin $(\mathrm{g} / \mathrm{dL}) \dagger$ & $1.1 \pm 0.2$ & $1.2(0.8-1.6)$ & $1.4 \pm 0.2$ & $1.5(1.1-1.7)$ & $1.3 \pm 0.2(1-1.7)$ \\
\hline Globulin (g/dL) & $2.0 \pm 0.4$ & $2.0(1.4-2.7)$ & $2.2 \pm 0.3$ & $2.2(1.6-2.6)$ & $2.1 \pm 0.3(1.5-2.7)$ \\
\hline$A: G$ ratio† & $0.6 \pm 0.08$ & $0.6(0.4-0.7)$ & $0.6 \pm 0.1$ & $0.6(0.5-0.8)$ & $0.6 \pm 0.1(0.5-0.7)$ \\
\hline $\operatorname{ALT}(\mathrm{U} / \mathrm{L})$ & $7.6 \pm 5.5$ & $7.0(1-25)$ & $14.6 \pm 16.0$ & $7.5(2-52)$ & $17 \pm 17(5-68)$ \\
\hline AST (U/L) & $322.5 \pm 135.9$ & $298.0(129-622)$ & $310.0 \pm 187.1$ & $237.0(105-760)$ & $356 \pm 156(88-669)$ \\
\hline ALP $(U / L) \dagger$ & $961.9 \pm 1,046.7$ & $527.0(25-3,43 I)$ & $317.7 \pm 334.9$ & $204.5(69-1516)$ & $262 \pm 120(99-463)$ \\
\hline $\mathrm{LDH}(\mathrm{U} / \mathrm{L})$ & $7,234.9 \pm 4,699.6$ & $5,754.0(1,938-16,922)$ & $6,453.7 \pm 5,643.8$ & $4,752.0(1,995-23,378)$ & $3,878 \pm 2,079(1,093-7,728)$ \\
\hline Cholesterol $(\mathrm{mg} / \mathrm{dL}) \dagger$ & $3 \mid 2.1 \pm 73.7$ & $307.0(177-462)$ & $204.8 \pm 43.0$ & $194.0(136-288)$ & $185 \pm 58(138-356)$ \\
\hline CK $(\mathrm{U} / \mathrm{L})$ & $6,932.9 \pm 8,064.3$ & $5,899.5(2|0-38,28|)$ & $3,749.0 \pm 3,246.2$ & $2,760.5(499-12,254)$ & $3,403 \pm 2,136(1,210-8,367)$ \\
\hline
\end{tabular}

*Reference data represent reported values for clinically convalescent, rehabilitated, cold-stunned Kemp's ridley sea turtles. ${ }^{13}$ †Convalescent values are significantly $(P<0.05)$ different from initial values. $¥$ Measured with a refractometer.

$-=$ Not available. $\mathrm{A}: \mathrm{G}=$ Albumin-to-globulin. $\mathrm{ALP}=$ Alkaline phosphatase. $\mathrm{ALT}=$ Alanine aminotransferase. $\mathrm{AST}=$ Aspartate aminotransferase. $\mathrm{CK}=$ Creatine kinase. $\mathrm{Cr}=$ Creatinine. $\mathrm{LDH}=$ Lactate dehydrogenase. $\mathrm{Na}: \mathrm{K}=$ Sodium-to-potassium. $\mathrm{TCO}_{2}=\mathrm{Total}_{\text {carbon dioxide. }}$ 
of the 8 individuals that received surgical treatment. Successfully rehabilitated individuals were hospitalized for a median of 256 days prior to release, during which time radiographic remodeling was observed (range, 133 to 552 days; mean \pm SD, $270 \pm 91$ days) .

Surviving sea turtles from which Enterococcus spp were recovered $(n=10)$ were hospitalized for a median of 256 days (range, 196 to 314 days; mean \pm SD, $253 \pm 33$ days), with radiographic lytic lesions detected after a mean of 48 days and a period of progression of 124 days (range 15 to 380). Surviving individuals from which $S$ marcescens was recovered $(\mathrm{n}=$ 8) were hospitalized for a median of 299 days (range, 232 to 552 days; mean \pm SD, $338 \pm 109$ days), with radiographic lytic lesions detected after a mean of 44 days of hospitalization and a period of lesion progression of 67 days (range, 42 to 112 days).

Three of the included sea turtles with osteolytic lesions died, each of complicated disease conditions. One sea turtle in which severe pneumonia and gastritis was diagnosed at admission was ultimately euthanized because of progressive pneumonia and osteomyelitis. Culture of bronchoalveolar lavage samples yielded Paecilomyces lilacinus (Purpureocillium lilacinum) and $M$ chelonae. In this individual, osteolytic lesions were present in the left shoulder joint and mandible. Bacterial culture of blood and surgical site swab samples yielded $E$ faecalis and Mycobacterium sp, respectively. Histologic evaluation revealed chronic granulomatous and fibrosing epiphyseal osteomyelitis accompanied by synovitis and myositis with intralesional acid-fast bacteria and granulomatous pneumonia with intralesional fungal hyphae.

The second sea turtle developed severe gastritis during hospitalization and was ultimately euthanized because of extensive gastric ulceration and necrosis. A focal osteolytic lesion was present in the right elbow joint, from which $E$ faecalis was recovered. Histologic evaluation revealed chronic perforating gastritis with secondary septic coelomitis and severe, chronic, granulomatous osteoarthritis and chondronecrosis of the shoulder joint with no intralesional pathogens.

The third turtle was euthanized because of its moribund condition, and ultimately severe bacterial and fungal sepsis was diagnosed (Figure 1). Epiphyseal osteolytic lesions were present in the left elbow, left hip, and right shoulder joints and bilaterally in the carpus and tarsus. Bacterial culture of blood and elbow joint samples yielded $S$ marcescens. Postmortem histologic evaluation revealed severe granulomatous coelomitis, epicarditis, hepatitis, nephritis, gastritis, osteomyelitis, and pneumonia with intralesional fungal hyphae (Metarrhizium sp).

\section{Discussion}

Osteolytic lesions were detected in approximately $1 \%$ of cold-stunned Kemp's ridley sea turtles that were hospitalized over an 11-year period, and most of these lesions were associated with bacterial osteomyelitis.
Although a small percentage of the caseload, such cases required substantial investment of diagnostic and therapeutic resources. On average after initial diagnosis, sea turtles required 213 days of hospitalization (range, 70 to 537 days), consistent with other reports of osteomyelitis in sea turtles. ${ }^{16,20}$ However, treatment of such cases of osteomyelitis was successful, with an $88 \%$ survival rate in the present study.

For most sea turtles, initial or empirical management with antimicrobial prophylaxis or an NSAID was ineffective, whereas more specific treatment based on microbial culture and antimicrobial susceptibility testing was often successful. Although all sea turtles received analgesics, the treatment plans differed among individuals and the effectiveness of specific analgesics could not be determined. No obvious improvement was noted with use of analgesics as part of the initial or empirical management. For sea turtles in which medical management did not result in resolution, surgical management was often successful. Surgical management allowed direct debridement of the joint, whereas systemic antimicrobial penetration of the joint may have been hindered by vascular alterations, ischemia, and necrotic bone. ${ }^{28,29}$ Although surgery was effective, surgical patients still required a mean of 162 days of further hospitalization prior to release. All 25 sea turtles required long-term management, with the duration of hospitalization varying with concurrent complications, such as pneumonia, sepsis, or gastroenteritis.

Some aspects of the pathogenesis of osteolytic lesions in cold-stunned sea turtles remain unclear. Lesions may occur secondary to immunosuppression and bacteremia or fungemia, with microbial seeding of devitalized bone subsequent to ischemia from hypothermia. Lesions could also be secondary to sterile necrosis of bone due to ischemic injury from hypothermia, as described in some humans with frostbite. ${ }^{14,30}$ Osteomyelitis complicated by sepsis was the most common finding among sea turtles of the present study, as evidenced by concurrent isolation of the same bacteria from blood and osteolytic site samples in 8 individuals, concurrent severe infections in others, predilection for subchondral lesions as observed in other species, ${ }^{31,32}$ and the presence of heterophilic and granulomatous osteomyelitis at the time of cytologic and histologic evaluation..$^{33}$ Similar lesions have been found in non-cold-stunned sea turtles with septicemia from traumatic injuries and other sources of infection. ${ }^{10,17,19,21}$ Recent or ongoing antimicrobial treatment, fastidious culture requirements, and chronicity may have affected culture results for those sea turtles with no microbial growth. Woven bone formation is a common feature of osteomyelitis in some other animals, ${ }^{34}$ but was not among sea turtles.

Decreased perfusion at low body temperatures could play a role in bone ischemia from hypothermia, although we would expect a predilection for this in the distal appendages and dermal bone (ie, skull and carapace) and, potentially, a higher prevalence of osteolytic lesions in sea turtles if hypothermic ischemia was the sole inciting cause. 
Radiographic changes in sea turtles typically occurred after approximately 2 months of hospitalization (mean, 55 days), suggesting that the onset of abnormal radiographic findings lags behind the initial insult, as noted in other species. ${ }^{32}$ While speculative, this lag time may be considerably longer in sea turtles than in mammals due to their slower metabolism. In most sea turtles, radiographic changes progressed in severity, characterized by a larger affected area or involvement of additional bones or joints. In our opinions, CT provided no advantages over conventional radiology for assessment of these lesions. However, in the few sea turtles in which it was performed, scintigraphy was useful in distinguishing active lesions from quiescent lesions and in case management decisions, as reported previously. ${ }^{14}$

Most blood and osteolytic site samples yielded bacterial growth; however, 6 sea turtles had negative culture results for site samples and 10 had negative results for blood samples. At the time of microbial culture, most sea turtles were receiving at least 1 antimicrobial. This treatment often consisted of a broad-spectrum antimicrobial for presumptive treatment of pneumonia, sepsis, or both. The preexisting antimicrobial treatment may have affected any potential bacterial growth in osteolytic lesions and resulted in a false negative culture result. In similar studies ${ }^{28,29}$ regarding septic arthritis in foals and calves, only $52 \%$ to $59 \%$ of joint aspirate samples yielded bacterial growth, despite visible bacteria on cytologic evaluation. False-negative culture results may have also been attributable to inadvertent sampling of adjacent sterile tissue rather than the intended osteolytic (infected) site. Sea turtles were considered for surgery if they had persistent positive culture results for fine-needle aspirate samples despite susceptibility-based antimicrobial treatment. Because culture results for intraoperative necrotic tissue samples were correlated with results for previous fineneedle aspirate samples, it may be more practical in certain cases to collect samples for microbial culture at the time of surgery because of increased accessibility of the affected site.

Bacteria isolated from blood and osteolytic site samples included $E$ faecalis, Enterococcus spp, $S$ marcescens, $M$ chelonae, Pseudomonas spp, $V$ alginolyticus, C braakii, and Acinetobacter sp, with a higher prevalence of Enterococcus spp and $S$ marcescens. Many of these species have been previously associated with infection in reptiles, ${ }^{35,36}$ and many can be cultured from the digestive tract and skin of apparently healthy reptiles. ${ }^{36}$ Septicemia and osteomyelitis associated with Enterococcus spp have been previously described in cold-stunned Kemp's ridley sea turtles, including several individuals that were also part of the present study. ${ }^{15}$ Serratia spp are opportunistic pathogens associated with septicemic cutaneous ulcer disease in freshwater chelonians and are thought to be normal oral flora in green sea turtles (Chelonia mydas). ${ }^{35-37}$

Mycobacterial infections have been previously described in sea turtles, often carrying a poor prog- nosis. ${ }^{38}$ Similarly, in the present study, the single sea turtle with a mycobacterial infection was euthanized after prolonged treatment because of persistent infection at multiple body sites. To the authors' knowledge, the successful treatment of deeply established mycobacterial infections in sea turtles has not been reported. The 3 sea turtles that died had concurrent diseases at the time of diagnosis and treatment for osteomyelitis, including severe gastritis, severe pneumonia, and systemic fungal infection. These comorbidities suggest the complexities that can be encountered during the medical management of coldstunned sea turtles.

Hematologic and plasma biochemical data indicated that the sea turtles of the present study were in fairly stable physiologic condition at the time of radiographic diagnosis. Although statistical differences were documented between the time of diagnosis and convalescence, many of the differences may have represented nonspecific changes that might occur during the course of convalescence of cold-stunned sea turtles. ${ }^{11,12}$ For example, in cold-stunned Kemp's ridley sea turtles, generalized physiologic stress, reduced food consumption, and generalized cellular injury may often result in initial leukocytosis, hyperglycemia, hypoalbuminemia, increased activities of tissue enzymes, and other nonspecific changes, which often resolve during convalescence. ${ }^{13}$ Although some of these changes (eg, leukocytosis, heterophilia, and high plasma alkaline phosphatase activity) could have been the result of osteomyelitis, additional comparative analyses would be required to determine whether any of the observed changes were specifically associated with the osteolytic lesions.

Because of the high incidence of bacterial pneumonia and other bacterial diseases in cold-stunned Kemp's ridley sea turtles, prophylactic antimicrobial treatment is often provided beginning on the first day of hospitalization. ${ }^{5}$ It is possible that antimicrobial selection at admission affected the results of microbial culture or may have selected for certain bacterial species. Historically, ceftazidime has been used as the initial antimicrobial for coldstunned sea turtles at New England Aquarium. However, concern about ceftazidime as a risk factor for Enterococcus infection led clinicians to adopt oxytetracycline as the initial antimicrobial in $2013 .{ }^{15,39,40}$ Indeed, in the present study, ceftazidime was used as the initial or subsequent antimicrobial for all 10 sea turtles from which Enterococcus spp were later isolated. For all 8 sea turtles from which $S$ marcescens was isolated, oxytetracycline had been used as the initial antimicrobial. These findings suggested that initial antimicrobial choice may influence the species of bacteria that later cause infection, with ceftazidime perhaps reducing the likelihood of gramnegative infection while increasing the risk of Enterococcus infection and oxytetracycline increasing the risk of gram-negative break-through infections, such as Serratia infections. Although the empirical use of antimicrobials for these cases remains justifiable, cli- 
nicians may choose to be more selective, monitoring closely for breakthrough infections regardless of the initial antimicrobial used.

In the present study, 25 cases of osteolytic lesions were documented during rehabilitation of cold-stunned Kemp's ridley sea turtles. Initial diagnosis was performed via radiography, followed by microbial culture, cytologic evaluation, and histologic evaluation of affected sites, characterizing the lesions as osteomyelitis, leading to septic arthritis in many cases. Affected sea turtles had concurrent clinical abnormalities that included localized swelling, lameness, lethargy, and hyporexia. Empirical medical management alone was ineffective in most cases, whereas antimicrobial use in accordance with susceptibility test results, surgical management, or both were overall effective for cases of persistent positive culture results for osteolytic sites. Clinicians should be aware of the possibility of osteomyelitis in debilitated sea turtles and should pursue additional diagnostic testing as indicated.

\section{Acknowledgments}

No third-party funding or support was received in connection with this study or the writing or publication of the manuscript. The authors declare that there were no conflicts of interest.

The authors thank Dr. Catherine Muckle and Dr. Shannon Martinson from the Department of Pathology and Microbiology at the Atlantic Veterinary College for their support and contributions in reviewing this manuscript as well as Adam Kennedy of the New England Aquarium for assistance with medical record data collection.

\section{References}

1. Wibbels T, Bevan E. Lepidochelys kempii: the IUCN Red List of Threatened Species 2019. Accessed September 1, 2020. https://www.iucnredlist.org/species/11533/155057916

2. Márquez-Millan R, Burchfield PM, Díaz-Flores J, Mejia MV. Status of the Kemp's ridley turtle, Lepidochelys kempii. Chelonian Conserv Biol. 2005;4(4):761-766.

3. Morreale SJ, Standora EA. Western North Atlantic waters: crucial developmental habitat for Kemp's ridley and loggerhead sea turtles. Chelonian Conserv Biol. 2005;4(4):872-882.

4. Morreale SJ, Meylan AB, Sadove SS, Standora EA. Annual occurrence and winter mortality of sea turtles in New York waters. J Herpetol. 1992;26(3):301-308.

5. Innis CJ, Staggs LA. Cold-stunning. In: Manire CA, Norton TM, Stacy BA, Norton T, Innis C, eds. Sea Turtle Health and Rehabilitation. J. Ross Publishing; 2017:675-687.

6. Wyneken J, Mader DR, Weber ES, et al. Medical care of sea turtles. In: Mader D, ed. Reptile Medicine and Surgery. 2nd ed. Elsevier; 2006:972-1007.

7. Gerle E, DiGiovanni R, Pisciotta R. A fifteen year review of cold-stunned sea turtles in New York waters. In: Proceedings of the 18th Annual International Symposium on Sea Turtle Biology and Conservation. National Oceanic and Atmospheric Administration; 2000:222-224.

8. Stacy NI, Innis CJ, Hernandez JA. Development and evaluation of three mortality prediction indices for cold-stunned Kemp's ridley sea turtles (Lepidochelys kempii). Conserv Physiol. 2013;1:cot003. doi: 10.1093/conphys/cot003

9. Rockwell KE, Innis CJ, Merigo C, Prescott R. The effect of delayed hospitalization in cold-stunned Kemp's ridley turtles (Lepidochelys kempii). J Herpetol Med Surg. 2017;27(3-4):93-96.

10. Cruciani B, Shneider $\mathrm{F}$, Ciccione $\mathrm{S}$, et al. Management of polyarthritis affecting sea turtles at Kélonia, the reunion island sea turtle observatory $(2013-17) . J$ Wildl Dis. 2019;55(2):455-461.
11. Innis C, Nyaoke AC, Williams R III, et al. Pathologic and parasitologic findings of cold-stunned Kemp's ridley sea turtles (Lepidochelys kempii) stranded on Cape Cod, Massachusetts, 2001-2006. J Wildl Dis. 2009;45(3):594-610.

12. Keller KA, Innis CJ, Tlusty MF, et al. Metabolic and respiratory derangements associated with death in cold-stunned Kemp's ridley sea turtles (Lepidochelys kempii): 32 cases (2005-2009). J Am Vet Med Assoc. 2012;240(3):317-323.

13. Innis CJ, Ravich JB, Tlusty MF, et al. Hematologic and plasma biochemical findings in cold-stunned Kemp's ridley turtles: 176 cases (2001-2005). J Am Vet Med Assoc. 2009;235(4):426-432.

14. Solano M, Innis C, Smith C, Ernest SW III. Scintigraphic and radiographic evaluation of appendicular skeletal lesions in cold-stunned Kemp's ridley sea turtles. Vet Radiol Ultrasound. 2008;49(4):388-394.

15. Innis CJ, Braverman H, Cavin JM, et al. Diagnosis and management of Enterococcus spp. infections during rehabilitation of cold-stunned Kemp's ridley turtles (Lepidochelys kempii): 50 cases (2006-2012). J Am Vet Med Assoc. 2014;245(3):315-323.

16. Greer LL, Strandberg JD, Whitaker BR. Mycobacterium chelonae osteoarthritis in a Kemp's Ridley sea turtle (Lepidochelys kempii).J Wildl Dis. 2003;39(3):736-741.

17. Guthrie A, George J, deMaar TW. Bilateral chronic shoulder infections in an adult green sea turtle (Chelonia mydas). J Herpetol Med Surg. 2010;20(4):105-108.

18. Ogden JA, Rhodin AGJ, Conlogue GJ, Light TR. Pathobiology of septic arthritis and contagious osteomyelitis in a leatherback turtle (Dermochelys coriacea). J Wildl Dis. $1981 ; 17(2): 277-287$.

19. Raidal SR, Shearer PL, Prince RIT. Chronic shoulder osteoarthritis in a loggerhead turtle (Caretta caretta). Aust Vet J. 2006;84(7):231-234.

20. Harms CA, Lewbart GA, Beasley J. Medical management of mixed nocardial and unidentified fungal osteomyelitis in a Kemp's Ridley sea turtle, Lepidochelys kempii. J Herpetol Med Surg. 2002;12(3):21-26.

21. Pace A, Meomartino L, Affuso A, Mennonna G, Hochsheid S, Dipineto L. Aeromonas induced polyostotic osteomyelitis in a juvenile loggerhead sea turtle (Caretta caretta). Dis Aquat Organ. 2018;132(1):79-84.

22. Thrall D. Radiographic features of bone tumors and bone infection. In: Thrall D, ed. Textbook of Veterinary Diagnostic Radiology. 6th ed. Elsevier; 2013:307-318.

23. Romer AS. Osteology of the Reptiles. Krieger Publishing; 1997.

24. Wyneken J. The anatomy of sea turtles. US Department of Commerce NOAA Technical Memorandum NMFS-SEFSC-2001-470. Southeast Fisheries Science Center; 2011:1172.

25. Snover ML, Rhodin AGJ. Comparative ontogenetic and phylogenetic aspects of chelonian chondro-osseous growth and skeletochronology. In: Wyneken J, Godfrey MH, Bels V, eds. Biology of Turtles. CRC Press; 2008:17-43.

26. Innis C, Merigo C, Dodge $\mathrm{K}$, et al. Health evaluation of leatherback turtles (Dermochelys coriacea) in the Northwestern Atlantic during direct capture and fisheries gear disentanglement. Chelonian Conserv Biol. 2010;9(2):205-222.

27. Clinical and Laboratory Standards Institute. Performance standards for antimicrobial susceptibility testing; twentieth informational supplement. M100-S20. Clinical and Laboratory Standards Institute; 2010:30(1).

28. Constant C, Nichols S, Desrochers A, et al. Clinical findings and diagnostic test results for calves with septic arthritis: 64 cases (2009-2014). J Am Vet Med Assoc. 2018;252(8):9951005.

29. Neil KM, Axon JE, Begg AP, et al. Retrospective study of 108 foals with septic osteomyelitis. Aust Vet J. 2010;88(1-2):4-12.

30. Selke AC Jr. Destruction of phalangeal epiphyses by frostbite. Radiology. 1969;93(4):859-860.

31. Gieling F, Peters S, Erichsen C, Richards RG, Zeiter S, Moriarty TF. Bacterial osteomyelitis in veterinary orthopaedics: pathophysiology, clinical presentation and advances in treatment across multiple species. Vet J. 2019;250:44-54.

32. Verschooten F, Vermeiren D, Devriese L. Bone infection 
in the bovine appendicular skeleton: a clinical, radiographic, and experimental study. Vet Radiol Ultrasound. 2000; $41(3): 250-260$.

33. Maxie MG. Bones and joints. In: Craig LE, Dittmer KE, Thompson KG, eds. Jubb, Kennedy \& Palmer's Pathology of Domestic Animals. 5th ed. Elsevier; 2007:97-101.

34. Zachary JF. Bones, joints, tendons, and ligaments. In: Olson EJ, Carlson CS, eds. In: Pathologic Basis of Veterinary Disease Consult. 6th ed. Elsevier; 2012:983-986.

35. Cooper JE. Dermatology. In: Mader D, ed. Reptile Medicine and Surgery. 2nd ed. Elsevier; 2006:972-1007.

36. Buller NB. Aquatic animal species and organism relation ship. In: Bacteria From Fish and Other Aquatic Animals: $A$ Practical Identification Manual. 2nd ed. CABI Publishing; 2004:1-75.

37. Santoro M, Hernández G, Caballero M. Aerobic bacterial flora of nesting green turtles (Chelonia mydas) from Tortuguero National Park, Costa Rica.J Zoo Wildl Med. 2006;37:549-552.

38. Innis C, Frasca S. Bacteria and fungi. In: Manire CA, Norton TM, Stacy BA, Norton T, Innis C, eds. Sea Turtle Health and Rehabilitation. J. Ross Publishing; 2017:779-790.

39. Harms CA, Papich MG, Stamper MA, Ross PM, Rodriguez MX, Hohn AA. Pharmacokinetics of oxytetracycline in loggerhead sea turtles (Caretta caretta) after single intravenous and intramuscular injections. J Zoo Wildl Med. 2004;35(4):477-488.

40. Hollenbeck BL, Rice LB. Intrinsic and acquired resistance mechanisms in enterococcus. Virulence. 2012;3(5):421-433.

\section{Supplementary Materials}

Supplementary materials are posted online at the journal website: avmajournals.avma.org 